\title{
Development and validation of a modified performance-oriented mobility assessment tool for assessing mobility in children with hypophosphatasia
}

\author{
Dawn Phillips $^{\mathrm{a}, 1, *}$, Donna Griffin ${ }^{\mathrm{b}}$, Tracy Przybylski ${ }^{\mathrm{b}}$, Erica Morrison ${ }^{\mathrm{b}}$, Amy L. Reeves $^{\mathrm{b}}$, Marc Vallee $^{\mathrm{c}}$, \\ Kenji P. Fujita ${ }^{\mathrm{d}}$ and Katherine L. Madson ${ }^{\mathrm{b}, 1}$ \\ ${ }^{a}$ Division of Physical Therapy, University of North Carolina, Chapel Hill, NC, USA \\ ${ }^{\mathrm{b}}$ Shriners Hospitals for Children, St. Louis, MO, USA \\ ${ }^{\mathrm{c}}$ Biostatistics, Alexion Pharmaceuticals, Inc., Boston, MA, USA \\ ${ }^{\mathrm{d}}$ Clinical Development, Alexion Pharmaceuticals, Inc., Boston, MA, USA
}

Accepted 1 June 2018

\begin{abstract}
.
PURPOSE: To modify the Performance-Oriented Mobility Assessment-Gait (POMA-G) subtest and validate this modified POMA-G (mPOMA-G) in children with hypophosphatasia (HPP), a rare metabolic disorder that can manifest with musculoskeletal symptoms that impair mobility and ambulation.

METHODS: Based on feedback from an expert panel, the POMA-G was modified by removing gait initiation/path assessments and expanding the rating scale for step length/continuity to capture aspects of observational gait analysis relevant to children with HPP. Three trained physical therapists used the mPOMA-G for video-based assessments of gait in 14 children with childhood HPP who participated in a clinical study of asfotase alfa or in a natural history study. Intraclass correlation coefficients (ICCs) were calculated to determine interrater and intrarater agreement. Concurrent validity was evaluated by correlations with other validated assessment tools.

RESULTS: Across 192 observations from available videos, interrater and intrarater agreement of mPOMA-G scores was significant (ICCs: 0.76 for both; $P<0.001$ ). mPOMA-G scores had strong concurrent validity with the Childhood Health Assessment Questionnaire, Pediatric Outcomes Data Collection Instrument Transfer and Mobility Scale, Sports and Physical Function subscale, and 6-Minute Walk Test (all $P \leqslant 0.0002$ ).

CONCLUSION: The mPOMA-G is a reliable and valid measure for detecting clinically significant impairments in children with HPP.
\end{abstract}

Keywords: Hypophosphatasia, pediatrics, early ambulation, mobility limitation, patient outcome assessment, validation studies

\footnotetext{
${ }^{1}$ Affiliation at the time the work was done.

* Corresponding author: Dawn Phillips, Division of Physical Therapy, University of North Carolina, 1104 Willow Drive, Chapel Hill, NC 27517, USA. Tel.: +1 919604 0840; E-mail: DawnPhillipspt @gmail.com.
}

\section{Introduction}

Hypophosphatasia (HPP) is a rare inherited systemic metabolic disorder caused by low tissue-nonspecific alkaline phosphatase activity [1-3]. Signs and symptoms of HPP can manifest in utero through adulthood and can vary widely $[1,3]$. In children, common

1874-5393/18/\$35.00 (C) 2018 - IOS Press and the authors. All rights reserved

This article is published online with Open Access and distributed under the terms of the Creative Commons Attribution Non-Commercial License (CC BY-NC 4.0). 
manifestations include radiographic evidence of abnormal mineralization in provisional zones of the long bones, knocked/bowed knees, muscle weakness, musculoskeletal pain, fractures, poor growth, and premature tooth loss with roots intact [1,4,5]. Musculoskeletal aspects of HPP can impair mobility and ambulation, which may have implications for activities of daily living, community participation, and quality of life.

The Performance-Oriented Mobility Assessment (POMA) is validated for evaluating gait and balance in elderly and community-dwelling adults [6]. The POMA gait subtest (POMA-G) contains components that can be applied directly or indirectly to measure gait impairments (e.g., trunk sway, walking stance, step continuity) in patients with HPP [6]. Although the POMA-G has also been used to differentiate gait characteristics in deaf versus hearing children aged $7-$ 17 years [7], it has not been validated for use in other pediatric conditions or in typically developing children. Further, some studies suggest that a ceiling effect exists with the POMA-G, limiting sensitivity to change in interventional studies [8,9].

With the approval of asfotase alfa, an enzymereplacement therapy for HPP [10-12], validated tools are needed for effective assessment and treatment of children with HPP. In the current study, the POMA-G tool was modified, and the modified version was validated for assessment of gait impairment in children with HPP

\section{Evaluation of the POMA-G and development of the MPOMA-G}

An expert panel of physicians, physical therapists, and statisticians evaluated the suitability of the POMA$\mathrm{G}$ for assessing gait impairment in children with HPP using observational, noninstrumented video footage. Most components of the POMA-G were relevant and could be reliably used. However, several modifications to the POMA-G were recommended by the expert panel to adapt it for use in children with HPP while preserving its clinimetric properties, resulting in the modified POMA-G (mPOMA-G) (Table 1). Modifications included: (1) removing the rating of initiation of gait, because children with HPP typically do not have difficulty initiating gait; (2) expanding the assessment of step length and step continuity from a 2- to 3-point scale to provide greater sensitivity and precision to detect change; (3) removing the rating of path, because children, whether or not they have HPP, may lack fo- cused, purposeful gait or may follow a divergent path, and because single-view video footage may not permit reliable assessment of path deviations; (4) adding new items within observations for step length and height; (5) clarifying descriptions of specific items to increase sensitivity and consistency among raters; and (6) creating a scoring key that provides detailed instructions on item rating and includes illustrations (Fig. 1) of step continuity and hip/knee flexion during swing for reference during rating $[13,14]$. The lowest score is 0 for each of the items, and the highest score is 1 or, for some observations, 2 (Table 1).

\section{3. mPOMA-G validation}

The study was conducted in accordance with the International Conference on Harmonisation Guideline for Good Clinical Practice after review and approval by the Washington University in St. Louis Institutional Review Board. Three physical therapists with experience evaluating patients with HPP used the MPOMA$G$ to score videos of 14 children with HPP while walking. Patients (age range: 5-15 years) were enrolled in an open-label asfotase alfa clinical study (NCT00952484) with extension (NCT01203826) or a natural history study (NCT02235493). Videos of children in the treated group $(n=8)$ were taken before treatment (at pre-enrollment time points $[n=5$; range: 12-36 months prior to baseline] and at baseline) and after treatment $(3,6,12,15,18,24,30$, and 42 months). Videos of individuals in the natural history group ( $n=$ 6) were taken at routine follow-up visits. Parents or legal guardians of the patients provided written informed consent and patients provided written assent before videotaping. Raters were not involved in treating patients receiving asfotase alfa and did not have access to identifiers and recording dates. Visible faces in videos were permanently blurred, and all videos $(n=64)$ were assigned a new masking code and randomized before each scoring.

All raters were trained on the scoring key and applied it to sample videos of normal and atypical gait patterns, including those exhibited by patients with HPP not in the study sample. Three median scores (one from each of the 3 raters) for each item were determined for each available time point and were summed to generate a $0-12$ overall score $(0=$ most disability to $12=$ no disability). To establish intrarater reliability, all videos were rescored by the same raters 8 months later. 
Table 1

The mPOMA-G Scale (modified POMA-G*)

\begin{tabular}{|c|c|c|}
\hline Observation & & Score \\
\hline \multirow[t]{14}{*}{ Step length and height } & Right swing foot & \\
\hline & - Does not pass the left stance foot with step & $=0$ \\
\hline & - Right heel passes the left stance foot & $=1$ \\
\hline & $\begin{array}{l}\text { - Right foot passes the left stance foot by at least the length of individual's foot between the stance toe } \\
\text { and swing heel }\end{array}$ & $=2$ \\
\hline & Right foot clear & \\
\hline & - Right foot does not clear floor completely with step or raises foot by more than $1-2$ inches & $=0$ \\
\hline & - Right foot completely clears floor & $=1$ \\
\hline & Left swing foot & \\
\hline & - Does not pass the right stance foot with step & $=0$ \\
\hline & - Left heel passes the right stance foot & $=1$ \\
\hline & $\begin{array}{l}\text { - Left foot passes the right stance foot by at least the length of individual's foot between the stance toe } \\
\text { and swing heel }\end{array}$ & $\equiv 2$ \\
\hline & Left foot clear & \\
\hline & - Left foot does not clear floor completely with step or raises foot by more than $1-2$ inches & $=0$ \\
\hline & - Left foot completely clears floor & $=1$ \\
\hline \multirow[t]{2}{*}{ Step symmetry } & - Right and left step length not equal (estimate) & $=0$ \\
\hline & - Right and left step appear equal & $=1$ \\
\hline \multirow[t]{5}{*}{ Step continuity } & - Stopping or discontinuity between steps & $=0$ \\
\hline & - Steps appear continuous unilaterally (observe raising heel of 1 foot as heel of other foot touches the & $\equiv 1$ \\
\hline & floor unilaterally) or flat foot contact on stance limb when heel of other foot touches the floor & \\
\hline & $\overline{\text { bilaterally, no breaks or stops in stride }}$ & \\
\hline & $\begin{array}{l}\text { - Steps appear continuous bilaterally (observe raising heel of } 1 \text { foot as heel of other foot touches the } \\
\text { floor, bilaterally), no breaks or stops in stride, step lengths equal }\end{array}$ & $\equiv 2$ \\
\hline \multirow[t]{4}{*}{ Trunk } & $\begin{array}{l}\text { - Marked sway or uses walking aid. Marked sway }=\text { moderate lateral flexion as the result of instability } \\
\text { bilateral or unilateral }\end{array}$ & $=0$ \\
\hline & - No marked sway but flexion of knees or back or spreads arms out while walking compensatory & $=1$ \\
\hline & $\begin{array}{l}\text { patterns, such as trunk flexion, knee flexion, arm abduction, or retraction to increase postural } \\
\text { stability while walking }\end{array}$ & \\
\hline & - No sway, no flexion, no use of arms, and no walking aid & $=2$ \\
\hline \multirow[t]{2}{*}{ Walk stance } & - Heels always apart, wide base of support utilized to increase postural stability & $=0$ \\
\hline & - Heels intermittently apart or almost touching while walking & $=1$ \\
\hline \multirow[t]{2}{*}{ Initiation of gait } & - Any hesitancy or multiple attempts & $=\mathbf{0}$ \\
\hline & - No hesitancy & $=\mathbf{1}$ \\
\hline \multirow[t]{3}{*}{ Path } & - Marked deviation & $=\mathbf{0}$ \\
\hline & - Mild/moderate deviation or uses walking aid & $=\mathbf{1}$ \\
\hline & - Straight without walking aid & $=2$ \\
\hline Gait score & & $/ 12$ \\
\hline
\end{tabular}

* Modification to the POMA-G included deleted items or deleted details in existing definitions (bold), new items (italics), and revisions that provide more detail to existing items (underlined). POMA-G = Performance-Oriented Mobility Assessment-Gait; mPOMA-G = modified Performance-Oriented Mobility Assessment-Gait.

All analyses were conducted using SAS software (Cary, NC). Interrater and intrarater agreement for scores was determined by calculating intraclass correlation coefficients (ICCs). For interrater ICC, a 2-way random-effects analysis of variance model was used, with terms for subject-visit, rater, and interaction between subject-visit and rater. For intrarater ICC, a 2way random-effects analysis of variance model was used, with terms for subject-visit-rater, review (original or retest), and interaction between subject-visitrater and review. The null hypothesis (ICC $\leqslant 0$ ) was tested at a 0.025 level of significance and rejected if the $P$ value was $\leqslant 0.025$ and ICC $>0$.

In total, 192 observations were completed by the 3 raters (17 observations per rater from videos of treated patients at historical pre-treatment time points and the natural history group at baseline and last assessment; 47 observations per rater from videos of treated patients at baseline and post-baseline time points). Both interrater and intrarater ICC was $0.76(P<0.0001$ and $P<0.001$, respectively).

For concurrent validation of mPOMA-G scores to other outcome measures assessing functional impairments, linear regressions were conducted and their corresponding Pearson correlation coefficients $(r)$ were obtained based on data from children with infantile $(n=5)$ and childhood HPP $(n=8)$ enrolled in the open-label asfotase alfa clinical study and extension. 


\section{A. Step Continuity}

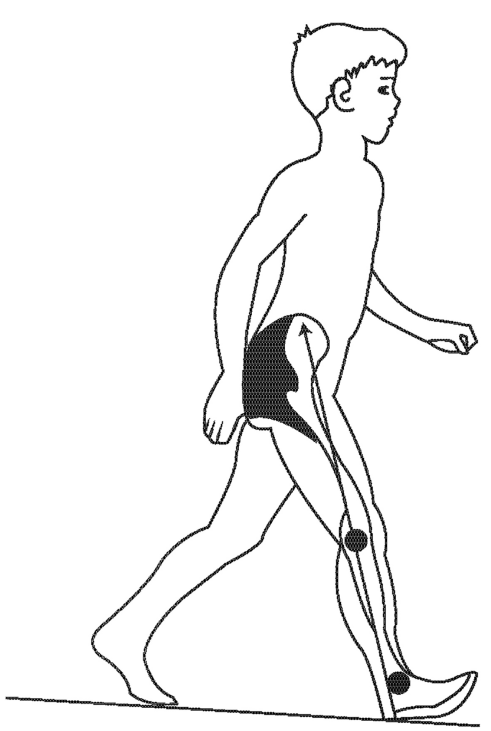

Adapted with permission from: Gage JR: An Overview of Normal Walking, in Greene WB (ed): Instructional Course Lectures 39. Rosemont, IL, American Academy of Orthopaedic Surgeons, 1990, pp 291-303

\section{B. Midswing-Foot Clearance}

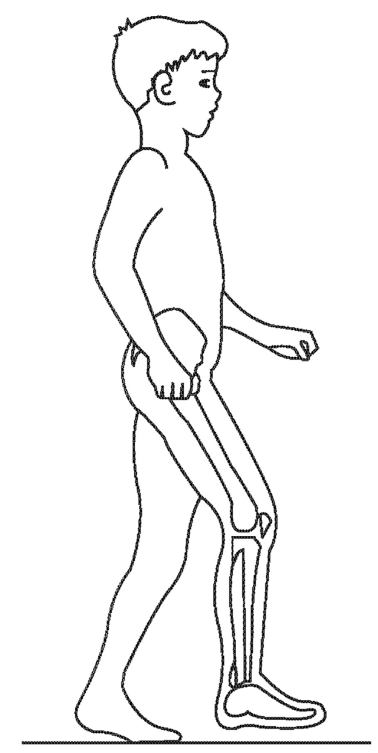

Adapted with permission from: Stout GL: Gait: Development and Analysis, in Campbell SK (ed) Physical Therapy for Children. Philadelphia, PA, WB Saunders, 1995, pp 79-104.

Fig. 1. Example of step continuity and hip/knee flexion during swing for reference during rating.

Pearson correlation coefficients for children with both infantile and childhood HPP demonstrated strong concurrent validity between mPOMA-G scores and the Childhood Health Assessment Questionnaire (CHAQ) Disability Index [15] (infantile HPP, $r=-0.86$; childhood HPP, $r=-0.72$ ), Pediatric Outcomes Data Collection Instrument (PODCI) [15] Transfer and Basic Mobility subscale (infantile HPP, $r=0.72$; childhood HPP, $r=0.57$ ) and Sports and Physical Function subscale (infantile HPP, $r=0.78$; childhood HPP, $r=$ $0.65)$, and meters walked on the 6-Minute Walk Test (6MWT) (infantile HPP, $r=0.83$; childhood HPP, $r=$ 0.70) (all $P \leqslant 0.0002$; Fig. 2).

\section{Discussion}

This analysis demonstrates that the mPOMA-G is a reliable and valid tool for detecting clinically significant impairments and changes in gait from videos of children with HPP. ICC calculations indicated good interrater and intrarater agreement. The mPOMA-G showed strong concurrent validity with other validated measures of disability, activities of daily living, and physical function, which is important given that chil- dren's usual daily activities and their performance in age-appropriate settings may be evaluated more effectively using $\geqslant 2$ outcome measures [15]. The CHAQ and PODCI have been used frequently in children and adolescents with other conditions that affect bone, muscle, and physical activity [15-18]. The PODCI and mPOMA-G association was stronger for PODCI Sports and Physical Function than Transfer and Basic Mobility, which measures shorter distance ambulation, such as climbing 1 flight of stairs and simple transfers, and had a ceiling effect for some patients with scores above the normative mean of 50 ( \pm 10$)$ [15,19]. The PODCI Sports and Physical Function contains items such as running a short distance, climbing 3 flights of stairs, walking 3 blocks, and participating in recreational outdoor activities and competitive sports. Step size and overall gait efficiency, measured by the mPOMA-G, may be functionally relevant to execution of these tasks and provide an indicator of ambulatory capacity in the school and community. Patient age should be considered when using the mPOMA and other mobility assessment tools. The patient must be able to follow instructions when performing mobility tests, and children aged $\leqslant 4$ years may be limited in this regard [20]. The mPOMA-G was 
(A) CHAQ Disability Index

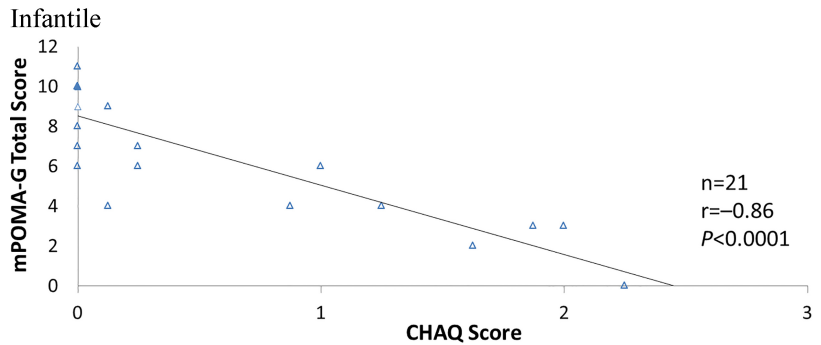

(B) PODCI Transfer and Basic Mobility

Infantile

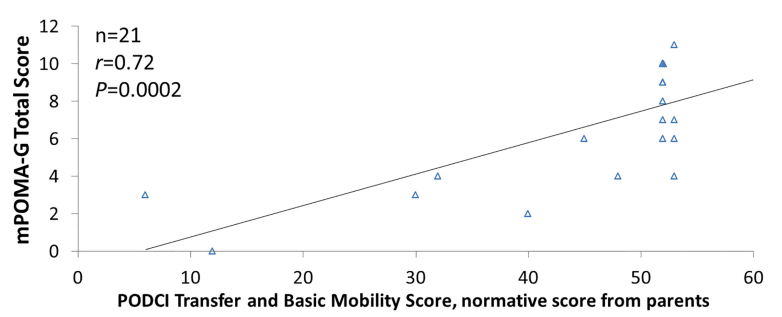

(C) PODCI Sports/Physical Fuctioning

Infantile

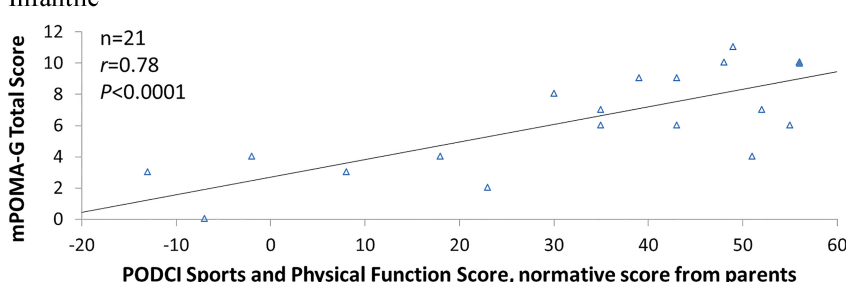

(D) 6MWT

Infantile

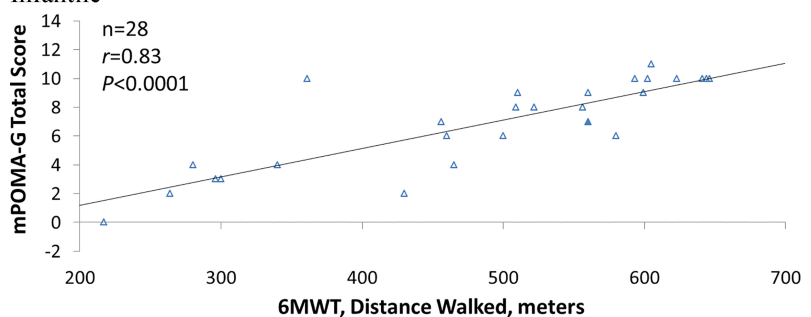

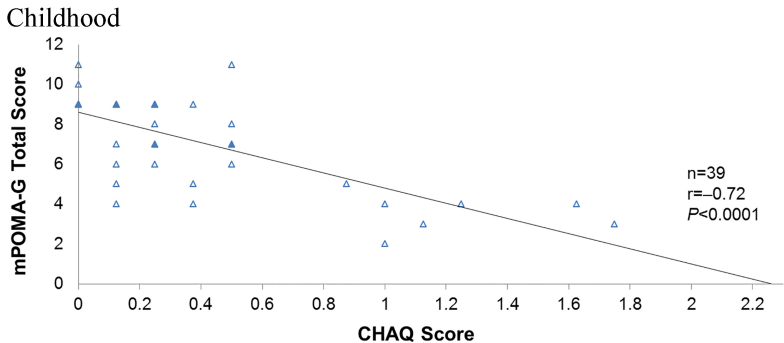
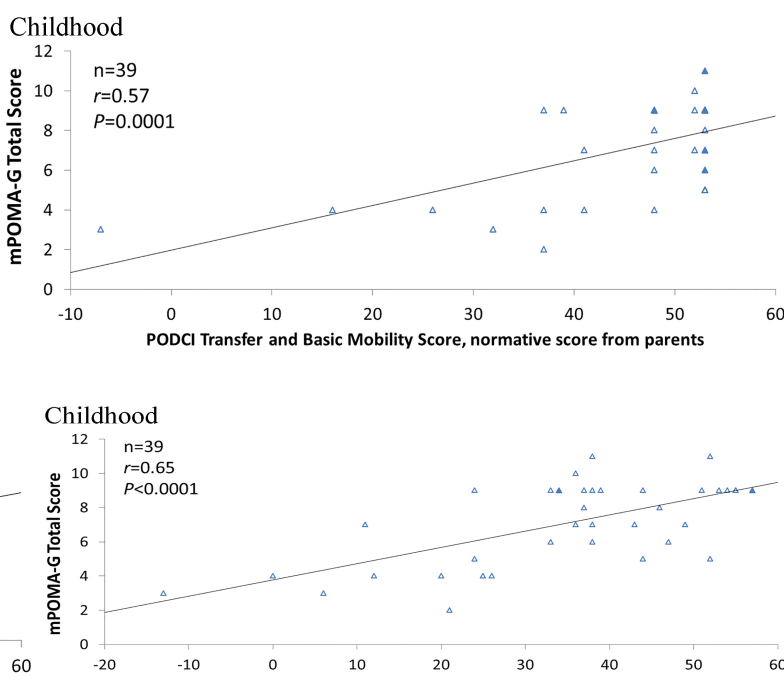

PODCI Sports and Physical Function Score, normative score from parents

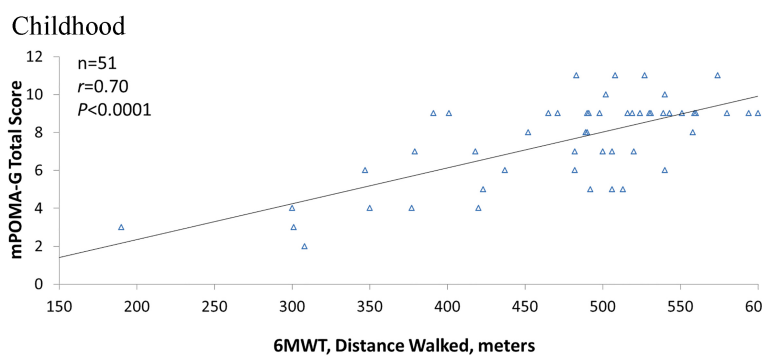

Fig. 2. Linear regression and Pearson correlation analyses of mPOMA-G with (A) CHAQ Disability Index; (B) PODCI Transfer and Basic Mobility Subscale, Normative Score from Parents; (C) PODCI Sports and Physical Function Subscale, Normative Score from Parents; and (D) 6MWT, Distance Walked in Children with Infantile and Childhood HPP. The $\mathrm{n}$ represents number of observations. Filled triangles indicate overlapping observations. 6MWT $=6$-Minute Walk Test; CHAQ $=$ Childhood Health Assessment Questionnaire; mPOMA-G $=$ modified Performance-Oriented Mobility Assessment-Gait; PODCI $=$ Pediatric Outcomes Data Collection Instrument; $\mathrm{r}=$ Pearson correlation coefficient.

validated in patients aged $\geqslant 5$ years, and the 6MWT was performed by patients aged $\geqslant 4$ years for assessing concurrent validity with the mPOMA-G. Future studies validating this instrument in typically developing children are warranted.

Current healthcare resource constraints limit availability of gait assessment laboratories and use of detailed gait assessment tools. mPOMA-G items were reliably evaluated in observational, noninstrumented video footage from clinical visits and from a singleview perspective of children with HPP performing the 6MWT. Video capture does not require the use of special equipment, advanced technical expertise, or a studio. Furthermore, in contrast to real-time subjective observations, the resulting video footage provides an objective record that can be replayed repeatedly and analyzed precisely.

To understand the impact of recent advances in the 
medical management of HPP on patients' quality of life, healthcare providers need tools that accurately and thoroughly assess mobility. The mPOMA-G is a reliable and valid clinical tool that can be easily administered in the clinical setting to assess gait impairment of patients with HPP.

\section{Acknowledgments}

This study was sponsored by Alexion Pharmaceuticals, Inc. Editorial support was provided by Bina J. Patel, PharmD, CMPP, of Peloton Advantage, LLC, and was funded by Alexion Pharmaceuticals, Inc.

\section{Conflict of interest}

DP was a consultant for Alexion Pharmaceuticals, Inc., at the time of the study and had received funding and travel support from Alexion for consulting and participating on advisory boards.

DG, TP, EM, and ALR have no conflict of interest to report.

$\mathrm{MV}$ and KPF are employees of and may own stock/ options in Alexion Pharmaceuticals, Inc., which sponsored the study.

KLM received speaker/consulting fees, research grants, and travel support from Alexion Pharmaceuticals, Inc.

\section{References}

[1] Whyte MP. Hypophosphatasia: nature's window on alkaline phosphatase function in man. In: Bilezikian JP, Raisz LG, Rodan GA, eds. Principles of Bone Biology. 3rd ed. San Diego, CA: Academic Press; 2008: pp. 1573-98.

[2] Weiss MJ, Cole DE, Ray K, Whyte MP, Lafferty MA, Mulivor RA, et al. A missense mutation in the human liver/ bone/kidney alkaline phosphatase gene causing a lethal form of hypophosphatasia. Proc Natl Acad Sci U S A. 1988; 85(20): 7666-9.

[3] Silver MM, Vilos GA, Milne KJ. Pulmonary hypoplasia in neonatal hypophosphatasia. Pediatr Pathol. 1988; 8(5): 48393.

[4] Whyte MP. Hypophosphatasia and how alkaline phosphatase promotes mineralization. In: Thakker RV, Whyte MP, Eisman JA, Igarashi T, eds. Genetics of Bone Biology and Skeletal Disease. 2nd edn. San Diego: Elsevier (Academic Press); 2018: pp. 581-504.
[5] Whyte MP. Physiological role of alkaline phosphatase explored in hypophosphatasia. Ann N Y Acad Sci. 2010; 1192: 190-200.

[6] Tinetti ME. Performance-oriented assessment of mobility problems in elderly patients. J Am Geriatr Soc. 1986; 34(2): 119-26.

[7] de Souza Melo R, da Silva PWA, Tassitano RM, Macky CFST, da Silva LVC. Balance and gait evaluation: comparative study between deaf and hearing students. Rev Paul Pediatr. 2012; 30(3): 385-91. doi: 10.1590/S0103-058220120003 00012.

[8] Faber MJ, Bosscher RJ, van Wieringen PC. Clinimetric properties of the performance-oriented mobility assessment. Phys Ther. 2006; 86(7): 944-54. doi: 10.1093/ptj/86.7.944.

[9] Shore WS, de Lateur BJ, Kuhlemeier KV, Imteyaz H, Rose G, Williams MA. A comparison of gait assessment methods: Tinetti and GAITRite electronic walkway [letter]. J Am Geriatr Soc. 2005; 53(11): 2044-5. doi: 10.1111/j.15325415.2005.00479_9.x.

[10] Whyte MP, Greenberg CR, Salman NJ, Bober MB, McAlister WH, Wenkert D, et al. Enzyme-replacement therapy in lifethreatening hypophosphatasia. N Engl J Med. 2012; 366(10): 904-13 doi: 10.1056/NEJMoa1106173.

[11] Strensiq [summary of product characteristics]. RueilMalmaison, France: Alexion Europe; 2017.

[12] Strensiq [package insert]. Boston, MA: Alexion Pharmaceuticals, Inc.; 2018.

[13] Gage JR. An overview of normal walking. Instr Course Lect. 1990; 39: 291-303.

[14] Stout JL. Gait: development and analysis. In: Campbell SK ed. Physical Therapy for Children. Philadelphia, PA: WB Saunders; 1995.

[15] Klepper SE. Measures of pediatric function: Child Health Assessment Questionnaire (C-HAQ), Juvenile Arthritis Functional Assessment Scale (JAFAS), Pediatric Outcomes Data Collection Instrument (PODCI), and Activities Scale for Kids (ASK). Arthritis Care Res. 2011; 63(Suppl 11): S371-82.

[16] Bayhan IA, Er MS, Nishnianidze T, Ditro C, Rogers KJ, Miller F, et al. Gait pattern and lower extremity alignment in children with diastrophic dysplasia. J Pediatr Orthop. 2016; 36(7): 709-14. doi: 10.1097/BPO.0000000000000530.

[17] Raluy-Callado M, Chen WH, Whiteman DA, Fang J, Wiklund I. The impact of Hunter syndrome (mucopolysaccharidosis type II) on health-related quality of life. Orphanet J Rare Dis. 2013; 8: 101. doi: 10.1186/1750-1172-8-101.

[18] Dempster H, Porepa M, Young N, Feldman BM. The clinical meaning of functional outcome scores in children with juvenile arthritis. Arthritis Rheum. 2001; 44(8): 1768-74. doi: 10. 1002/1529-0131(200108)44:8<1768::AID-ART312>3.0.CO; 2-Q.

[19] American Academy of Orthopaedic Surgeons, Pediatric Orthopaedic Society of North America, American Academy of Pediatrics, Shriner's Hospitals. Pediatric Outcomes Questionnaire. Available at: https://www.aaos.org/research/outcomes/ pediatric.pdf. Accessed July 15, 2017.

[20] Geiger R, Strasak A, Treml B, Gasser K, Kleinsasser A, Fischer V, et al. Six-minute walk test in children and adolescents. J Pediatr. 2007; 150(4): 395-9. doi: 10.1016/j.jpeds.2006.12. 052 . 